\title{
A LEVEL-RANK DUALITY FOR PARAFERMION VERTEX OPERATOR ALGEBRAS OF TYPE A
}

\author{
CHING HUNG LAM \\ (Communicated by Kailash C. Misra)
}

\begin{abstract}
We show that the tensor product of the parafermion vertex operator algebras $K\left(s l_{k+1}, n+1\right) \otimes K\left(s l_{n+1}, k+1\right)$ can be embedded as a full subVOA into the lattice VOA $V_{A_{n} \otimes A_{k}}$. The decomposition of $V_{A_{n} \otimes A_{k}}$ as a direct sum of irreducible $K\left(s l_{k+1}, n+1\right) \otimes K\left(s l_{n+1}, k+1\right)$-modules is also obtained. In addition, we show that the parafermion VOA $K\left(s l_{n}, k\right)$ contains a full subVOA isomorphic to a tensor product of $W$-algebras $W_{s l_{k}}(1,1) \otimes$ $W_{s l_{k}}(1,2) \otimes \cdots \otimes W_{s l_{k}}(1, n-1)$.
\end{abstract}

\section{INTRODUCTION}

Let $\mathfrak{g}$ be a finite dimensional simple Lie algebra and $\widehat{\mathfrak{g}}$ the affine Kac-Moody Lie algebra associated with $\mathfrak{g}$. Let $L_{\widehat{\mathfrak{g}}}(k, 0)$ be the irreducible vacuum module of $\hat{\mathfrak{g}}$ with level $k$, where $k$ is a positive integer. The vertex operator algebra $L_{\widehat{\mathfrak{g}}}(k, 0)$ contains a Heisenberg vertex operator algebra corresponding to a Cartan subalgebra $\mathfrak{h}$ of $\mathfrak{g}$. The commutant $K(\mathfrak{g}, k)$ of the Heisenberg vertex operator algebra in $L_{\widehat{\mathfrak{g}}}(k, 0)$ is called a parafermion vertex operator algebra (see [2 [5]).

In this article, we study parafermion vertex operator algebras associated to the Lie algebra $s l_{n}(\mathbb{C})$. We notice that there is an interesting duality between the parafermion vertex operator algebra $K\left(s l_{k+1}, n+1\right)$ and $K\left(s l_{n+1}, k+1\right)$. Namely, we show that the tensor product $K\left(s l_{k+1}, n+1\right) \otimes K\left(s l_{n+1}, k+1\right)$ can be embedded as a full subVOA into the lattice VOA $V_{A_{n} \otimes A_{k}}$, where $n$ and $k$ are positive integers. Recall that the tensor product $A \otimes B$ of two integral lattices $A$ and $B$ is defined to be the integral lattice which is isomorphic to $A \otimes_{\mathbb{Z}} B$ as a $\mathbb{Z}$-module and has the inner product given by

$$
\left(\alpha \otimes \beta, \alpha^{\prime} \otimes \beta^{\prime}\right)=\left(\alpha, \alpha^{\prime}\right)_{A} \cdot\left(\beta, \beta^{\prime}\right)_{B},
$$

for any $\alpha, \alpha^{\prime} \in A$, and $\beta, \beta^{\prime} \in B$ (see [9]). The decomposition of $V_{A_{n} \otimes A_{k}}$ as a sum of irreducible $K\left(s l_{k+1}, n+1\right) \otimes K\left(s l_{n+1}, k+1\right)$-modules is also obtained. The main theorem is as follows. (See Section 3.2 for the definitions of $\mathcal{Y}_{n+1, k+1}^{\text {aff }}(0), \pi(Y)$ and $\pi\left({ }^{t} Y\right)$.)

Theorem 1.1. The lattice VOA $V_{A_{n} \otimes A_{k}}$ contains a full subalgebra isomorphic to $K\left(s l_{k+1}, n+1\right) \otimes K\left(s l_{n+1}, k+1\right)$. Moreover, we have the decomposition

$$
V_{A_{n} \otimes A_{k}}=\bigoplus_{Y \in \mathcal{Y}_{n+1, k+1}^{a f f}(0)} K_{s l_{n+1}, k+1}(\pi(Y), 0) \otimes K_{s l_{k+1}, n+1}\left(\pi\left({ }^{t} Y\right), 0\right) .
$$

Received by the editors October 3, 2012 and, in revised form, February 6, 2013.

2010 Mathematics Subject Classification. Primary 17B69.

This work was partially supported by NSC grant 100-2628-M-001005-MY4, Taiwan. 
The result above can be viewed as a parafermion version of the well-known conformal embedding of $L_{\hat{s l}_{n+1}}(k+1,0) \otimes L_{\hat{s l}_{k+1}}(n+1,0)$ into $V_{A_{n k+n+k}}$, 1, 10, 13 . In fact, the embedding of $K\left(s l_{k+1}, n+1\right) \otimes K\left(s l_{n+1}, k+1\right)$ into $V_{A_{n} \otimes A_{k}}$ can be deduced directly from the embedding of $L_{\hat{s} l_{n+1}}(k+1,0) \otimes L_{\hat{s} l_{k+1}}(n+1,0)$ into $V_{A_{n k+n+k}}$ also. Our method stresses the properties of certain integral lattices, which may provide some different viewpoints. In particular, we obtain an interesting relation between $K\left(s l_{n}, k\right)$ and certain $W$-algebras. Namely, we show that $K\left(s l_{n}, k\right)$ contains a full subalgebra isomorphic to a tensor product of $W$-algebras $W_{s l_{k}}(1,1) \otimes$ $W_{s l_{k}}(1,2) \otimes \cdots \otimes W_{s l_{k}}(1, n-1)$, where $W_{s l_{k}}(1, j)$ are some $W$-algebras constructed using coset constructions (see Section 5).

The organization of this article is as follows. In Section 2, we review the construction of parafermion vertex operator algebras and some of their modules. In Section 3, we study several sublattices of the root lattice $A_{(n+1)(k+1)-1}$. We also discuss the embedding of the affine VOA $L_{\hat{s} l_{n+1}}(k+1,0)$ and $L_{\hat{s} l_{k+1}}(n+1,0)$ into the lattice VOA $V_{A_{(n+1)(k+1)-1}}$. In Section 4 , we show that the VOA $K\left(s l_{k+1}, n+1\right) \otimes K\left(s l_{n+1}, k+1\right)$ can be embedded as a full subVOA into the lattice VOA $V_{A_{n} \otimes A_{k}}$. The decomposition of $V_{A_{n} \otimes A_{k}}$ as a direct sum of irreducible $K\left(s l_{k+1}, n+1\right) \otimes K\left(s l_{n+1}, k+1\right)$-modules is also determined. In Section 5 , we review a coset construction of certain $W$-algebras. The main result is an embedding of the tensor product $W_{s l_{k}}(1,1) \otimes W_{s l_{k}}(1,1) \otimes \cdots \otimes W_{s l_{k}}(1, n-1)$ into the parafermion VOA $K\left(s l_{n}, k\right)$.

\section{PARAFERmion Vertex operator Algebras}

In this section, we review the construction of parafermion vertex operator algebras and some of their modules [4, 5].

Let $\mathfrak{g}$ be a finite dimensional simple Lie algebra and $\widehat{\mathfrak{g}}$ the affine Kac-Moody Lie algebra associated with $\mathfrak{g}$. Let $\Pi=\left\{\alpha_{1}, \ldots, \alpha_{n}\right\}$ be a set of simple roots and $\theta$ the highest root. Let $Q$ be the root lattice of $\mathfrak{g}$ and $P=\left\{\Lambda \in \mathbb{Q} \otimes_{\mathbb{Z}} Q \mid\left\langle\alpha_{i}, \Lambda\right\rangle \in\right.$ $\mathbb{Z}$ for all $i=1, \ldots, n\}$ the weight lattice. For any positive integer $k$, we denote by

$$
P_{+}^{k}(\mathfrak{g})=\left\{\Lambda \in P \mid\left\langle\alpha_{i}, \Lambda\right\rangle \in \mathbb{Z}_{\geq 0} \text { for all } i=1, \ldots, n \text { and }\langle\theta, \Lambda\rangle \leq k\right\}
$$

the set of dominant integral weights for $\mathfrak{g}$ with level $k$.

Let $L_{\widehat{\mathfrak{g}}}(k, \Lambda)$ be the irreducible module of $\hat{\mathfrak{g}}$ with highest weight $\Lambda$ and level $k$. Then $L_{\widehat{\mathfrak{g}}}(k, 0)$ forms a simple VOA with the Virasoro element given by the Sugawara construction

$$
\Omega_{\mathfrak{g}, k}=\frac{1}{2\left(k+h^{\vee}\right)} \sum\left(u_{i}\right)_{-1} u^{i}
$$

where $h^{\vee}$ is the dual Coxeter number, $\left\{u_{i}\right\}$ is a basis of $\mathfrak{g}$ and $\left\{u^{i}:=\left(u_{i}\right)^{*}\right\}$ is the dual basis of $\left\{u_{i}\right\}$ with respect to the normalized Killing form (see [7]). Moreover, the central charge of $L_{\widehat{\mathfrak{g}}}(k, 0)$ is

$$
\frac{k \operatorname{dim} \mathfrak{g}}{k+h^{\vee}} .
$$

The vertex operator algebra $L_{\widehat{\mathfrak{g}}}(k, 0)$ contains a Heisenberg vertex operator algebra corresponding to a Cartan subalgebra $\mathfrak{h}$ of $\mathfrak{g}$. Let $M_{\hat{\mathfrak{h}}}(k, 0)$ be the vertex operator subalgebra of $L_{\widehat{\mathfrak{g}}}(k, 0)$ generated by $h(-1) \cdot \mathbf{1}$ for $h \in \mathfrak{h}$. The commutant $K(\mathfrak{g}, k)$ of $M_{\hat{\mathfrak{h}}}(k, 0)$ in $L_{\widehat{\mathfrak{g}}}(k, 0)$ is called a parafermion vertex operator algebra. 
The VOA $L_{\widehat{\mathfrak{g}}}(k, 0)$ is completely reducible as an $M_{\hat{\mathfrak{h}}}(k, 0)$-module, and we have a decomposition (see [5]).

Lemma 2.1. For any $\lambda \in \mathfrak{h}^{*}$, let $M_{\hat{\mathfrak{h}}}(k, \lambda)$ be the irreducible highest weight module for $\hat{\mathfrak{h}}$ with a highest weight vector $v_{\lambda}$ such that $h(0) v_{\lambda}=\lambda(h) v_{\lambda}$ for $h \in \mathfrak{h}$. Denote

$$
K_{\mathfrak{g}, k}(\lambda)=K_{\mathfrak{g}, k}(0, \lambda)=\left\{v \in L_{\widehat{\mathfrak{g}}}(k, 0) \mid h(m) v=\lambda(h) \delta_{m, 0} v \text { for } h \in \mathfrak{h}, m \geq 0\right\} .
$$

Then we have

$$
L_{\widehat{\mathfrak{g}}}(k, 0)=\bigoplus_{\lambda \in Q} K_{\mathfrak{g}, k}(\lambda) \otimes M_{\hat{\mathfrak{h}}}(k, \lambda),
$$

where $Q$ is the root lattice of $\mathfrak{g}$.

Similarly, for any $\Lambda \in P_{+}^{k}(\mathfrak{g})$, we also have the decomposition.

Lemma 2.2. Denote $K_{\mathfrak{g}, k}(\Lambda, \lambda)=\left\{v \in L_{\widehat{\mathfrak{g}}}(k, \Lambda) \mid h(m) v=\lambda(h) \delta_{m, 0} v\right.$ for $h \in$ $\mathfrak{h}, m \geq 0\}$. Then

$$
L_{\widehat{\mathfrak{g}}}(k, \Lambda)=\bigoplus_{\lambda \in \Lambda+Q} K_{\mathfrak{g}, k}(\Lambda, \lambda) \otimes M_{\hat{\mathfrak{h}}}(k, \lambda) .
$$

Remark 2.3. By definition, it is clear that $K_{\mathfrak{g}, k}(\Lambda, \lambda)$ is a VOA module of $K(\mathfrak{g}, k)$ for any $\Lambda$ and $\lambda$.

\section{Some sublattices of $A_{(n+1)(k+1)-1}$}

In this section, we consider several sublattices of the root lattice $A_{(n+1)(k+1)-1}$. Let $n, k$ be positive integers and $Z=\mathbb{Z}^{(n+1)(k+1)}$. Let

$$
L=\left\{\left(a_{1}, \ldots, a_{(n+1)(k+1)}\right) \in \mathbb{Z}^{(n+1)(k+1)} \mid \sum_{i=1}^{(n+1)(k+1)} a_{i}=0\right\} .
$$

Then we have $L \cong A_{(n+1)(k+1)-1}$.

Notation 3.1. Let $\mu_{n+1}: \mathbb{Z}^{k+1} \rightarrow \mathbb{Z}^{(n+1)(k+1)}$ and $d_{k+1}: \mathbb{Z}^{n+1} \rightarrow \mathbb{Z}^{(n+1)(k+1)}$ be defined by

$$
\mu_{n+1}\left(a_{1}, \ldots, a_{k+1}\right)=\left(a_{1}, \ldots, a_{1}, a_{2}, \ldots, a_{2}, \ldots, a_{k+1}, \ldots, a_{k+1}\right)
$$

and

$$
d_{k+1}\left(a_{1}, \ldots, a_{n+1}\right)=\left(a_{1}, \ldots, a_{n+1}, a_{1}, \ldots, a_{n+1}, \ldots, a_{1}, \ldots, a_{n+1}\right) .
$$

We also consider the injective maps $\eta_{i}: \mathbb{Z}^{n+1} \rightarrow \mathbb{Z}^{(n+1)(k+1)}$ and $\iota_{i}: \mathbb{Z}^{k+1} \rightarrow$ $\mathbb{Z}^{(n+1)(k+1)}$ defined by

$$
\eta_{i}\left(e_{j}\right)=e_{(n+1)(i-1)+j} \quad \text { and } \quad \iota_{i}\left(e_{j}\right)=e_{(n+1)(j-1)+i},
$$

for $i=1, \ldots, n+1, j=1, \ldots, k+1$, where $e_{i}$ is a row vector whose $i$-th entry is 1 and all other entries are zero.

Let $K^{1}=\mu_{n+1}\left(A_{k}\right)$ and $K^{2}=d_{k+1}\left(A_{n}\right)$. Then $K^{1} \cong \sqrt{n+1} A_{k}$ and $K^{2} \cong$ $\sqrt{k+1} A_{n}$. Moreover, it is easy to show that $K^{1}$ is orthogonal to $K^{2}$.

Definition 3.2. Let $L$ be an integral lattice and $X$ a sublattice. The sublattice

$$
A n n_{L}(X)=\{x \in L \mid\langle x, y\rangle=0 \text { for all } y \in X\}
$$

is called the annihilator of $X$ in $L$.

Lemma 3.3. We have $A n n_{L}\left(K^{1}\right) \cong A_{n}^{k+1}$ and $A n n_{L}\left(K^{2}\right) \cong A_{k}^{n+1}$. 
Proof. First we note that $A n n_{\mathbb{Z}^{n+1}}((1, \ldots, 1)) \cong A_{n}$. Thus,

$$
A n n_{L}\left(K^{1}\right)=A n n_{L}\left(\mu_{n+1}\left(A_{k}\right)\right)=\bigoplus_{i=1}^{k+1} \eta_{i}\left(A_{n}\right) \cong A_{n}^{k+1} \text {. }
$$

Similarly, we also have

$$
A n n_{L}\left(K^{2}\right)=A n n_{L}\left(d_{k+1}\left(A_{n}\right)\right)=\bigoplus_{i=1}^{n+1} \iota_{i}\left(A_{k}\right) \cong A_{k}^{n+1},
$$

as desired.

Remark 3.4. Since $K^{1}$ is orthogonal to $K^{2}$, we have $K^{2} \subset A n n_{L}\left(K^{1}\right)$ and $K^{1} \subset$ $A n n_{L}\left(K^{2}\right)$.

Notation 3.5. Set $M=A n n_{L}\left(K^{1}\right) \cong A_{n}^{k+1}$ and $N=A n n_{L}\left(K^{2}\right) \cong A_{k}^{n+1}$.

The following lemma can be proved easily by a simple calculation.

Lemma 3.6. Let $X=A n n_{L}\left(K^{1} \perp K^{2}\right)$. Then $X=A n n_{M}\left(K^{2}\right)=A n n_{N}\left(K^{1}\right) \cong$ $A_{k} \otimes A_{n}$.

3.1. Affine VOA. Next we recall a construction of affine VOA from [4. Let $\mathfrak{g}$ be a simple Lie algebra of $A, D, E$ type. Let $\mathfrak{h}$ be a Cartan subalgebra of $\mathfrak{g}$ and let $\Delta$ and $\Delta^{+}$be the sets of roots and positive roots. Then we have the root space decomposition

$$
\mathfrak{g}=\mathfrak{h} \oplus \bigoplus_{\alpha \in \Delta} \mathbb{C} x_{\alpha}
$$

Let $Q$ be the root lattice of $\mathfrak{g}$. We shall identify $\mathfrak{h}$ with $\mathbb{C} \otimes_{\mathbb{Z}} Q$. Set

$$
L_{0}=Q_{1} \oplus \cdots \oplus Q_{\ell},
$$

where each $Q_{i}$ is a copy of $Q$. For $\alpha \in Q$ and $h \in \mathfrak{h}$, we use $\alpha_{i}$ and $h_{i}$ to denote the corresponding elements in $Q_{i}$ and $\mathbb{C} \otimes Q_{i}$, respectively. Let $\hat{L}_{0}$ be a central extension of $L_{0}$ by $\langle\kappa\rangle$ where $\kappa^{2}=1$, and let $\iota: \mathbb{C}\left[L_{0}\right] \rightarrow \mathbb{C}\left\{L_{0}\right\}=\mathbb{C}\left[\hat{L}_{0}\right] /\langle\kappa+1\rangle$ be a linear isomorphism where $\mathbb{C}[G]=\left\{e^{g} \mid g \in G\right\}$ denotes the group algebra of a group $G$.

Let $V_{L_{0}}=M(1) \otimes \mathbb{C}\left\{L_{0}\right\}$ be the lattice VOA associated to $L_{0}[6]$. Then one has a linear injection $i$ from $\mathfrak{g}$ to $V_{L_{0}}$ as follows:

$$
\begin{aligned}
i(h) & =h_{1}(-1)+\cdots+h_{\ell}(-1) \in V_{L_{0}} \text { for } h \in \mathfrak{h}, \\
i\left(x_{\alpha}\right) & =\iota\left(e^{\alpha_{1}}\right)+\cdots+\iota\left(e^{\alpha_{\ell}}\right) \in V_{L_{0}} \text { for } \alpha \in \Delta .
\end{aligned}
$$

For $x \in \mathfrak{g}$, set

$$
x(z):=\sum_{n \in \mathbb{Z}}\left(x \otimes t^{n}\right) z^{-n-1} .
$$

Proposition 3.7 (4, Prop. 13.1]). The linear map $\pi: \widehat{\mathfrak{g}} \rightarrow \operatorname{End} V_{L_{0}}$ given by

$$
\begin{aligned}
\pi(c) & =\ell, \\
\pi(x(z)) & =Y(i(x), z) \quad \text { for } x \in \mathfrak{g}
\end{aligned}
$$

defines a $\widehat{\mathfrak{g}}$-module structure on $V_{L_{0}}$, of level $\ell$. Moreover, $V_{L_{0}}$ is completely reducible under $\widehat{\mathfrak{g}}$, and every irreducible component is a standard $\widehat{\mathfrak{g}}$-module of level $\ell$. 
Proposition $3.8([4])$. Let $U$ be the subVOA generated by $i(h)$ and $i\left(x_{\alpha}\right)$ for $h \in \mathfrak{h}$ and $\alpha \in \Delta$. Then $U$ is an irreducible integrable highest weight $\widehat{\mathfrak{g}}$-module of level $\ell$ and $U \cong L_{\widehat{\mathfrak{g}}}(\ell, 0)$ as a $V O A$.

Next, we study several Lie subalgebras in $\left(V_{L}\right)_{1}$. Let $M=\bigoplus_{i=1}^{k+1} \eta_{i}\left(A_{n}\right)$. For each positive root $\alpha \in \Delta^{+}\left(A_{n}\right)$, we define

$$
H_{\alpha}=d_{k+1}(\alpha)(-1) \cdot \mathbf{1}, \quad E_{\alpha}=\sum_{i=1}^{k+1} e^{\eta_{i}(\alpha)}, \quad F_{\alpha}=\sum_{i=1}^{k+1} e^{-\eta_{i}(\alpha)} .
$$

Then $\left\{H_{\alpha}, E_{\alpha}, F_{\alpha} \mid \alpha \in \Delta^{+}\left(A_{n}\right)\right\}$ spans a Lie subalgebra $s l_{n+1}(\mathbb{C})$ in $\left(V_{M}\right)_{1}$ and it also generates a subVOA isomorphic to $L_{\hat{s} l_{n+1}}(k+1,0)$ in $V_{M} \subset V_{L}$ [4, Chapter 14].

Similarly, for each positive root $\beta \in \Delta^{+}\left(A_{k}\right)$, we define

$$
H_{\beta}^{\prime}=\mu_{n+1}(\beta)(-1) \cdot \mathbf{1}, \quad E_{\beta}^{\prime}=\sum_{i=1}^{n+1} e^{\iota_{i}(\beta)}, \quad F_{\beta}^{\prime}=\sum_{i=1}^{n+1} e^{-\iota_{i}(\beta)}
$$

in $\left(V_{N}\right)_{1}$, where $N=\bigoplus_{i=1}^{n+1} \iota_{i}\left(A_{k}\right)$. In this case, $\left\{H_{\beta}^{\prime}, E_{\beta}^{\prime}, F_{\beta}^{\prime} \mid \beta \in \Delta^{+}\left(A_{k}\right)\right\}$ generates a subVOA isomorphic to $L_{\hat{s l}_{k+1}}(n+1,0)$ in $V_{N} \subset V_{L}$.

Proposition 3.9. The lattice VOA $V_{A_{n k+n+k}}$ contains a full subVOA isomorphic to the tensor product $L_{\hat{s l}_{n+1}}(k+1,0) \otimes L_{\hat{s}_{k+1}}(n+1,0)$.

Proof. By (2.2), the central charges of $L_{\hat{s l}_{n+1}}(k+1,0)$ and $L_{\hat{s} l_{k+1}}(n+1,0)$ are

$$
\frac{(k+1) n(n+2)}{k+1+n+1} \text { and } \frac{(n+1) k(k+2)}{n+1+k+1},
$$

respectively. The sum is thus equal to $(n+1)(k+1)-1$, which is the central charge of $V_{A_{(n+1)(k+1)-1}}$. Therefore, it suffices to show that $L_{\hat{s} l_{n+1}}(k+1,0)$ and $L_{\hat{s l}_{k+1}}(n+1,0)$ are mutually commutative in $V_{A_{n k+n+k}}$.

By definition, it is clear that $\left(H_{\alpha}\right)_{n} E_{\beta}^{\prime}=0,\left(H_{\alpha}\right)_{n} F_{\beta}^{\prime}=0$ for $n>0$ and

$$
\left(H_{\alpha}\right)_{0} E_{\beta}^{\prime}=\sum_{i=1}^{n+1}\left\langle d_{k+1}(\alpha), \iota_{i}(\beta)\right\rangle e^{\iota_{i}(\beta)} \text { and }\left(H_{\alpha}\right)_{0} F_{\beta}^{\prime}=\sum_{i=1}^{n+1}\left\langle d_{k+1}(\alpha),-\iota_{i}(\beta)\right\rangle e^{-\iota_{i}(\beta)}
$$

for any $\alpha \in \Delta^{+}\left(A_{n}\right)$ and $\beta \in \Delta^{+}\left(A_{k}\right)$. Since $\left\langle d_{k+1}(\alpha), \iota_{i}(\beta)\right\rangle=0$ for all $\alpha \in$ $\Delta^{+}\left(A_{n}\right)$ and $\beta \in \Delta^{+}\left(A_{k}\right)$, we have $\left(H_{\alpha}\right)_{0} E_{\beta}^{\prime}=0$ and $\left(H_{\alpha}\right)_{0} F_{\beta}^{\prime}=0$. Similarly, we also have $\left(H_{\beta}^{\prime}\right)_{0} E_{\alpha}=0$ and $\left(H_{\beta}^{\prime}\right)_{0} F_{\alpha}=0$.

Again, it is clear that $\left(E_{\alpha}\right)_{n}\left(E_{\beta}^{\prime}\right)=0,\left(E_{\alpha}\right)_{n}\left(F_{\beta}^{\prime}\right)=0,\left(F_{\alpha}\right)_{n}\left(E_{\beta}^{\prime}\right)=0$ and $\left(F_{\alpha}\right)_{n}\left(F_{\beta}^{\prime}\right)=0$ for $n>0$.

Let $\alpha=e_{i}-e_{j}$ and $\beta=e_{i^{\prime}}-e_{j^{\prime}}$ for $1 \leq i<j \leq n+1$ and $1 \leq i^{\prime}<j^{\prime} \leq k+1$. For each $1 \leq \ell \leq n+1$ and $1 \leq r \leq k+1$, the vector $\eta_{\ell}\left(e_{i}-e_{j}\right)+\iota_{r}\left(e_{i^{\prime}}-e_{j^{\prime}}\right)$ has norm 2 if and only if (1) $\ell=i^{\prime}$ and $j=r$ or (2) $\ell=j^{\prime}$ and $r=i$. Thus, we have

$$
\begin{aligned}
\left(E_{\alpha}\right)_{0}\left(E_{\beta}^{\prime}\right) & =\sum_{\ell, r}\left(e^{\eta_{\ell}\left(e_{i}-e_{j}\right)}\right)_{0} e^{\iota_{r}\left(e_{i^{\prime}}-e_{j^{\prime}}\right)} \\
& =\left(e^{\eta_{i^{\prime}}\left(e_{i}-e_{j}\right)}\right)_{0} e^{\iota_{j}\left(e_{i^{\prime}}-e_{j^{\prime}}\right)}+\left(e^{\eta_{j^{\prime}}\left(e_{i}-e_{j}\right)}\right)_{0} e^{\iota_{i}\left(e_{i^{\prime}}-e_{j^{\prime}}\right)} \\
& =e^{\eta_{i^{\prime}}\left(e_{i}\right)-\iota_{j}\left(e_{j^{\prime}}\right)}-e^{\iota_{i}\left(e_{i^{\prime}}\right)-\eta_{j^{\prime}}\left(e_{j}\right)}=0 .
\end{aligned}
$$

Note that $\left(e^{\eta_{j^{\prime}}\left(e_{i}-e_{j}\right)}\right)_{0} e^{\iota_{i}\left(e_{i^{\prime}}-e_{j^{\prime}}\right)}=-\left(e^{\iota_{i}\left(e_{i^{\prime}}-e_{j^{\prime}}\right)}\right)_{0} e^{\eta_{j^{\prime}}\left(e_{i}-e_{j}\right)}=-e^{\iota_{i}\left(e_{i^{\prime}}\right)-\eta_{j^{\prime}}\left(e_{j}\right)}$ and $\eta_{i^{\prime}}\left(e_{i}\right)=\iota_{i}\left(e_{i^{\prime}}\right)=e_{(n+1)\left(i^{\prime}-1\right)+i}$ (see Notation 3.1). 
Similarly, we also have $\left(E_{\alpha}\right)_{0}\left(F_{\beta}^{\prime}\right)=0,\left(F_{\alpha}\right)_{0}\left(E_{\beta}^{\prime}\right)=0$ and $\left(F_{\alpha}\right)_{0}\left(F_{\beta}^{\prime}\right)=0$ for any $\alpha \in \Delta^{+}\left(A_{n}\right)$ and $\beta \in \Delta^{+}\left(A_{k}\right)$. Since $L_{\hat{s} l_{n+1}}(k+1,0)$ and $L_{\hat{s} l_{k+1}}(n+1,0)$ are generated by $\left\{H_{\alpha}, E_{\alpha}, F_{\alpha} \mid \alpha \in \Delta^{+}\left(A_{n}\right)\right\}$ and $\left\{H_{\beta}^{\prime}, E_{\beta}^{\prime}, F_{\beta}^{\prime} \mid \beta \in \Delta^{+}\left(A_{k}\right)\right\}$ respectively, we have the desired conclusion.

Remark 3.10. The embedding of $L_{\hat{s l}_{n+1}}(k+1,0) \otimes L_{\hat{s} l_{k+1}}(n+1,0)$ in $V_{A_{n k+n+k}}$ is actually well-known in the literature (see for example [1,10, 12,13]).

3.2. Decomposition of $V_{A_{(n+1)(k+1)-1}}$. Next we discuss the decomposition of $V_{A_{(n+1)(k+1)-1}}$ as a module of $L_{\hat{s l}_{n+1}}(k+1,0) \otimes L_{\hat{s}_{k+1}}(n+1,0)$ (see [1, 10, 12, 14]).

First we recall a parametrization of the highest weights of $s l_{r}(\mathbb{C})$ using Young diagrams [14. Let $\left\{\lambda_{1}, \ldots, \lambda_{n}\right\}$ be a set of fundamental weights for $s l_{r}(\mathbb{C})$. Given an integer $l \geq 1$, let

$$
P_{+}^{l}(r)=\left\{\lambda=\sum_{i=1}^{r-1} a_{i} \lambda_{i} \mid \sum_{i=1}^{r-1} a_{i} \leq l, a \geq 0, a_{i} \in \mathbb{Z}\right\}
$$

be the set of dominant integral weights for $s l_{r}(\mathbb{C})$ of level $l$.

Definition 3.11 (see [14]). Given two positive integers $r$ and $l$, a Young diagram of type $(r, l)$ is a decreasing sequence of $r$ positive integers:

$$
Y=\left(y_{1} \geq y_{2} \geq \cdots \geq y_{r-1} \geq y_{r}\right) \text { such that } y_{1}-y_{r} \leq l .
$$

The set of Young diagrams of type $(r, l)$ is denoted by $\mathcal{Y}_{r, l}$.

We can think of a Young diagram of type $(r, l)$ as a collection of $r$ rows, where we put into the $i$-th row $y_{i}$ boxes. There is a natural map from $\mathcal{Y}_{r, l}$ to the set of dominant weights of $\operatorname{sl}(r)$ :

$$
\begin{aligned}
\pi: \mathcal{Y}_{r, l} & \rightarrow P_{+}^{l}(r) \\
Y & \mapsto \pi(Y)=\sum_{i=1}^{r-1}\left(y_{i}-y_{i+1}\right) \lambda_{i} .
\end{aligned}
$$

Define two finite subsets:

$$
\mathcal{Y}_{r, l}^{a f f}=\left\{Y \in \mathcal{Y}_{r, l} \mid y_{r} \leq l-1\right\} \quad \text { and } \quad \mathcal{Y}_{r, l}^{\text {fin }}=\left\{Y \in \mathcal{Y}_{r, l}^{a f f} \mid y_{1} \leq l\right\} .
$$

Note that $\pi\left(\mathcal{Y}_{r, l}^{a f f}\right)=P_{+}^{l}(r)$ and the map $\pi: \mathcal{Y}_{r, l}^{a f f} \rightarrow P_{+}^{l}(r)$ is $l$ to 1 since $y_{r} \leq l-1$.

Next we define a "transpose" map as follows (cf. [14]).

For $Y \in \mathcal{Y}_{r, l}^{\text {fin }}$, we have $y_{1} \leq l$ and $y_{r} \leq l-1$. Then the transpose of $Y$ is a Young diagram of size $(l, r)$. We define ${ }^{t} Y \in \mathcal{Y}_{l, r}^{\text {fin }}$ to be the transpose of $Y$, i.e., by putting $y_{i}$ boxes into the $i$-th column.

For $Y \in \mathcal{Y}_{r, l}^{a f f} \backslash \mathcal{Y}_{r, l}^{\text {fin }}$, we have $l+1 \leq y_{1} \leq 2 l-1$ and $y_{r} \leq l-1$. The Young diagram $Y$ decomposes as a union of two diagrams $Y_{1} \cup Y_{2}$ with $Y_{i} \in \mathcal{Y}_{r, l}^{\text {fin }}$, where the first one, $Y_{1}$, has in its $i$-th row $\min \left(l, y_{i}\right)$ boxes and the second one, $Y_{2}$, has in its $i$-th row $\max \left(y_{i}-l, 0\right)$ boxes. We define the transpose ${ }^{t} Y$ to be the union of the transposes ${ }^{t} Y_{1} \cup{ }^{t} Y_{2}$. Note that the map $Y \rightarrow{ }^{t} Y$ preserves the subsets $\mathcal{Y}_{r, l}^{\text {fin }} \subset \mathcal{Y}_{r, l}^{\text {aff }}$ and $\mathcal{Y}_{l, r}^{\text {fin }} \subset \mathcal{Y}_{l, r}^{\text {aff }}$. Note also the equalities

$$
\left|\mathcal{Y}_{r, l}^{\text {aff }}\right|=\left|\mathcal{Y}_{l, r}^{\text {aff }}\right|=l\left|P_{+}^{l}(r)\right|=r\left|P_{+}^{r}(l)\right| .
$$


Definition 3.12. Given a Young diagram $Y \in \mathcal{Y}_{r, l}$, we define its size

$$
|Y|=\sum_{i=1}^{r} y_{i} \quad \bmod r l
$$

It is clear from the definition that $\left|{ }^{t} Y\right|=|Y|$. We denote by $\mathcal{Y}_{r, l}^{f i n}(\lambda)$ and $\mathcal{Y}_{r, l}^{\text {aff }}(\lambda)$ the corresponding subsets of Young diagrams of size $\lambda$. Note that we identify $\mathbb{Z} / r l \mathbb{Z}=\{0, \ldots, r l-1\}$ so that $|Y| \in\{0, \ldots, r l-1\}$.

The next proposition can be found in [14] (see also [1, 10]).

Proposition 3.13. As a module of $L_{\hat{s} l_{n+1}}(k+1,0) \otimes L_{\hat{s l}_{k+1}}(n+1,0)$, the lattice VOA $V_{A_{n k+n+k}}$ is decomposed as

$$
V_{A_{n k+n+k}}=\bigoplus_{Y \in \mathcal{Y}_{n+1, k+1}^{a f f}(0)} L_{\hat{s l}_{n+1}}(k+1, \pi(Y)) \otimes L_{\hat{s}_{k+1}}\left(n+1, \pi\left({ }^{t} Y\right)\right) .
$$

Remark 3.14. We shall note that $\pi(Y) \in Q\left(A_{n}\right)$ (and $\pi\left({ }^{t} Y\right) \in Q\left(A_{k}\right)$ ) for any $Y \in \mathcal{Y}_{n+1, k+1}^{a f f}(0)$ (see [1]), where $Q\left(A_{n}\right)=A_{n}$ denotes the root lattice of type $A_{n}$.

$$
\text { 4. Parafermion VOAs } K_{s l_{n+1}}(k+1,0) \text { AND } K_{s l_{k+1}}(n+1,0)
$$

Next we discuss a construction of the parafermion VOA $K_{s l_{n}}(k, 0)$ from [4].

Recall from Section 3.1 that the elements

$$
H_{\alpha}=d_{k+1}(\alpha)(-1) \cdot \mathbf{1}, \quad E_{\alpha}=\sum_{i=1}^{k+1} e^{\eta_{i}(\alpha)}, \quad F_{\alpha}=\sum_{i=1}^{k+1} e^{-\eta_{i}(\alpha)}, \alpha \in \Delta^{+}\left(A_{n}\right),
$$

generate a simple VOA $L_{\hat{s l}_{n+1}}(k+1,0)$ inside $V_{L}$. Moreover,

$\left(E_{\alpha}\right)_{-1}^{k} E_{\alpha}=\left(\sum_{i=1}^{k+1} e^{\eta_{i}(\alpha)}\right)_{-1}^{k}\left(\sum_{i=1}^{k+1} e^{\eta_{i}(\alpha)}\right)=(k+1) ! e^{\eta_{1}(\alpha)+\eta_{2}(\alpha)+\cdots+\eta_{k+1}(\alpha)}=e^{d_{k+1}(\alpha)}$.

Thus, $e^{d_{k+1}(\alpha)} \in L_{\hat{s} l_{n+1}}(k+1,0)$ and similarly $e^{-d_{k+1}(\alpha)} \in L_{\hat{s l}_{n+1}}(k+1,0)$. Therefore, we may consider $V_{K^{2}}=V_{d_{k+1}\left(A_{n}\right)}$ as a subVOA of $L_{\hat{s} l_{n+1}}(k+1,0)$.

Similarly,

$$
H_{\beta}^{\prime}=\mu_{n+1}(\beta)(-1) \cdot \mathbf{1}, \quad E_{\beta}^{\prime}=\sum_{i=1}^{n+1} e^{\iota_{i}(\beta)}, \quad F_{\alpha}^{\prime}=\sum_{i=1}^{n+1} e^{-\iota_{i}(\beta)}
$$

generate a subVOA $L_{\hat{s} l_{k+1}}(n+1,0)$ in $V_{L}$, and we can also view $V_{K^{1}}=V_{\mu_{n+1}\left(A_{k}\right)}$ as a subVOA of $L_{\hat{s} l_{k+1}}(n+1,0)$.

Lemma 4.1. Let $\mathfrak{H}$ and $\mathfrak{H}^{\prime}$ be Lie subalgebras spanned by $\left\{H_{\alpha} \mid \alpha \in \Delta^{+}\left(A_{n}\right)\right\}$ and $\left\{H_{\beta}^{\prime} \mid \beta \in \Delta^{+}\left(A_{k}\right)\right\}$, respectively. Then we have

$$
\begin{aligned}
& K\left(s l_{n+1}, k+1\right)=\operatorname{Com}_{L_{\hat{s} l_{n+1}}(k+1,0)}\left(M_{\hat{\mathfrak{H}}}(k+1,0)\right)=\operatorname{Com}_{L_{\hat{s} l_{n+1}}(k+1,0)}\left(V_{K^{2}}\right), \\
& K\left(s l_{k+1}, n+1\right)=\operatorname{Com}_{L_{\hat{s} l_{k+1}}(n+1,0)}\left(M_{\hat{\mathfrak{H}}^{\prime}}(n+1,0)\right)=\operatorname{Com}_{L_{\hat{s} l_{k+1}}(n+1,0)}\left(V_{K^{1}}\right) .
\end{aligned}
$$

Remark 4.2. Recall that $K^{2}=d_{k+1}\left(A_{n}\right) \cong \sqrt{k+1} A_{n}$. For any $\beta \in Q\left(A_{n}\right)=A_{n}$, we have

$$
H_{\alpha}(0)\left(e^{\frac{1}{k+1} d_{k+1}(\beta)}\right)=\frac{1}{k+1}\left\langle d_{k+1}(\alpha), d_{k+1}(\beta)\right\rangle e^{\frac{1}{k+1} d_{k+1}(\beta)}=\langle\alpha, \beta\rangle e^{\frac{1}{k+1} d_{k+1}(\beta)} .
$$


That means $e^{\frac{1}{k+1} d_{k+1}(\beta)}$ is a highest weight vector for $\mathfrak{H}$ with the weight $\beta$. Similarly, $e^{\frac{1}{n+1} d_{n+1}\left(\beta^{\prime}\right)}$ is a highest weight vector for $\mathfrak{H}^{\prime}$ with the weight $\beta^{\prime}$ for any $\beta^{\prime} \in A_{k}$.

We shall identify $\sqrt{k+1} A_{n}$ with $K^{2}, \frac{1}{\sqrt{k+1}} A_{n}$ with $\frac{1}{k+1} K^{2}, \sqrt{n+1} A_{k}$ with $K^{1}$ and $\frac{1}{\sqrt{n+1}} A_{k}$ with $\frac{1}{n+1} K^{1}$. Then by Lemmas 2.1 and 2.2 , we have the following lemma.

Lemma 4.3. Let $\Lambda \in P_{+}^{k+1}(n+1)$. Then

$$
L_{\hat{s} l_{n+1}}(k+1,0)=\bigoplus_{\lambda \in \frac{1}{\sqrt{k+1}} A_{n} / \sqrt{k+1} A_{n}} K_{s l_{n+1}, k+1}(\sqrt{k+1} \lambda) \otimes V_{\lambda+\sqrt{k+1}} A_{n}
$$

and

$$
L_{\hat{s} l_{n+1}}(k+1, \Lambda)=\bigoplus_{\lambda \in \frac{1}{\sqrt{k+1}}\left(\Lambda+A_{n}\right) / \sqrt{k+1} A_{n}} K_{s l_{n+1}, k+1}(\Lambda, \sqrt{k+1} \lambda) \otimes V_{\lambda+\sqrt{k+1}} A_{n} .
$$

Proof. Since

$$
V_{\lambda+\sqrt{k+1} A_{n}}=\bigoplus_{\lambda^{\prime} \in \lambda+\sqrt{k+1} A_{n}} M(1) \otimes \mathbb{C} e^{\lambda^{\prime}}=\bigoplus_{\lambda^{\prime} \in \lambda+\sqrt{k+1} A_{n}} M_{\hat{\mathfrak{H}}}\left(k+1, \sqrt{k+1} \lambda^{\prime}\right),
$$

we have $K_{s l_{n+1}, k+1}(\Lambda, \sqrt{k+1} \lambda) \cong K_{s l_{n+1}, k+1}\left(\Lambda, \sqrt{k+1} \lambda^{\prime}\right)$ for any $\lambda^{\prime} \in \lambda+\sqrt{k+1} A_{n}$. The result now follows from Lemmas 2.1 and 2.2. Note that $\sqrt{k+1} \lambda, \sqrt{k+1} \lambda^{\prime} \in$ $\Lambda+A_{n}$.

Remark 4.4. It is shown in [4, Theorem 14.20] that $K_{s l_{n+1}, k+1}(\Lambda, \sqrt{k+1} \lambda)$, for $\Lambda \in P_{+}^{k+1}(n+1), \lambda \in\left(\sqrt{k+1} A_{n}\right)^{*}$, are irreducible $K\left(s l_{n+1}, k+1\right)$-modules.

Recall from Notation 3.5 that $M=A n n_{L}\left(K^{1}\right) \cong A_{n}^{k+1}, N=A n n_{L}\left(K^{2}\right) \cong A_{k}^{n+1}$ and $X=\operatorname{Ann}_{L}\left(K^{1} \perp K^{2}\right) \cong A_{n} \otimes A_{k}$. By taking the commutant, we have the proposition.

Proposition 4.5. The lattice VOA $V_{A_{n}^{k+1}}$ contains a full subVOA isomorphic to a tensor product of VOAs $L_{\hat{s l}_{n+1}}(k+1,0) \otimes K\left(s l_{k+1}, n+1\right)$. Moreover,

$$
V_{A_{n}^{k+1}}=\bigoplus_{Y \in \mathcal{Y}_{n+1, k+1}^{a f f}(0)} L_{\hat{s l}_{n+1}}(k+1, \pi(Y)) \otimes K_{s l_{k+1}, n+1}\left(\pi\left({ }^{t} Y\right), 0\right) .
$$

Proof. Since $M=A n n_{L}\left(K^{1}\right) \cong A_{n}^{k+1}$, we have

$$
\begin{aligned}
V_{A_{n}^{k+1}} & =\operatorname{Com}_{V_{L}}\left(V_{K}^{1}\right) \\
& =\bigoplus_{Y \in \mathcal{Y}_{n+1, k+1}^{a f f}(0)} L_{\hat{s l}_{n+1}}(k+1, \pi(Y)) \otimes K_{s l_{k+1}, n+1}\left(\pi\left({ }^{t} Y\right), 0\right)
\end{aligned}
$$

by Proposition 3.13 and Lemma 4.3. Note that

$$
K\left(s l_{k+1}, n+1\right)=\operatorname{Com}_{L_{\hat{s} l_{k+1}}}(n+1,0)\left(V_{K^{1}}\right)
$$

(see Lemma 4.1).

Similarly, we also have our main theorem by Proposition 3.13 and Lemmas 4.1 and 4.3 
Theorem 4.6. The lattice VOA $V_{A_{n} \otimes A_{k}}$ contains a full subVOA isomorphic to a tensor product of parafermion VOAs $K\left(s l_{n+1}, k+1\right) \otimes K\left(s l_{k+1}, n+1\right)$. Moreover, we have the decomposition

$$
V_{A_{n} \otimes A_{k}}=\bigoplus_{Y \in \mathcal{Y}_{n+1, k+1}^{a f f}(0)} K_{s l_{n+1}, k+1}(\pi(Y), 0) \otimes K_{s l_{k+1}, n+1}\left(\pi\left({ }^{t} Y\right), 0\right) .
$$

\section{Coset construction of Some $W$-Algebras}

In this section, we review a coset construction for certain $W$-algebras [8, 11]. We shall also show that the parafermion VOA $K\left(s l_{k+1}, n+1\right)$ naturally contains a tensor product of $W$-algebras as a full subVOA.

Let $\mathfrak{g}$ be a finite dimensional simple Lie algebra. Let $\hat{\mathfrak{g}}=\mathfrak{g} \otimes \mathbb{C}\left[t, t^{-1}\right] \oplus \mathbb{C} c \oplus \mathbb{C} d$ be the corresponding affine Lie algebra. For any $\hat{\mathfrak{g}}$-module $M, x \in \hat{\mathfrak{g}}$ and $m \in \mathbb{Z}$, we denote the action of $x \otimes t^{m}$ on $M$ by $x(m)$ and identify $\mathfrak{g} \otimes t^{0}$ with $\mathfrak{g}$.

Let $L_{\hat{\mathfrak{g}}}(1, \Lambda)$ and $L_{\hat{\mathfrak{g}}}\left(k, \Lambda^{\prime}\right)$ be two integrable highest weight representations of $\hat{\mathfrak{g}}$ with level 1 and $k$ respectively. Then $\hat{\mathfrak{g}} \oplus \hat{\mathfrak{g}}$ acts on the tensor product $L_{\hat{\mathfrak{g}}}(1, \Lambda) \otimes$ $L_{\hat{\mathfrak{g}}}\left(k, \Lambda^{\prime}\right)$ by

$$
(x(m) \oplus y(n))(v \otimes w)=(x(m) v) \otimes w+v \otimes(y(n) w),
$$

for any $x(n), y(m) \in \hat{\mathfrak{g}}$ and $v \otimes w \in L_{\hat{\mathfrak{g}}}(1, \Lambda) \otimes L_{\hat{\mathfrak{g}}}\left(k, \Lambda^{\prime}\right)$. On the other hand, $\hat{\mathfrak{g}}$ acts on $L_{\hat{\mathfrak{g}}}(1, \Lambda) \otimes L_{\hat{\mathfrak{g}}}\left(k, \Lambda^{\prime}\right)$ by the diagonal action

$$
x(n)(v \otimes w)=(x(n) v) \otimes w+v \otimes(x(n) w),
$$

for any $x(n) \in \hat{\mathfrak{g}}$ and $v \otimes w \in L_{\hat{\mathfrak{g}}}(1, \Lambda) \otimes L_{\hat{\mathfrak{g}}}\left(k, \Lambda^{\prime}\right)$. This gives a level $k+1$ representation $L_{\hat{\mathfrak{g}}}\left(k+1, \Lambda+\Lambda^{\prime}\right)$ of $\hat{\mathfrak{g}}$.

Definition 5.1. Let $U \cong L_{\hat{\mathfrak{g}}}(k+1,0)$ be the subVOA of $L_{\hat{\mathfrak{g}}}(1,0) \otimes L_{\hat{\mathfrak{g}}}(k, 0)$ induced by the diagonal action of $\hat{\mathfrak{g}}$. We define

$$
W_{\mathfrak{g}}(1, k)=\operatorname{Com}_{L_{\hat{\mathfrak{g}}}(1,0) \otimes L_{\hat{\mathfrak{g}}}(k, 0)}(U) .
$$

Note that the central charge of $W_{\mathfrak{g}}(1, k)$ is given by

$$
c=\frac{\operatorname{dim} \mathfrak{g}}{1+h^{\vee}}+\frac{k \operatorname{dim} \mathfrak{g}}{k+h^{\vee}}-\frac{(k+1) \operatorname{dim} \mathfrak{g}}{k+1+h^{\vee}}=\frac{k\left(k+1+2 h^{\vee}\right)}{\left(1+h^{\vee}\right)\left(k+h^{\vee}\right)\left(k+1+h^{\vee}\right)} \operatorname{dim} \mathfrak{g} .
$$

Remark 5.2. When $\mathfrak{g}=s l_{2}(\mathbb{C})$, it is known that $W_{s l_{2}}(1, k)$ is isomorphic to the simple Virasoro VOA $L\left(c_{k}, 0\right)$, where $c_{k}=1-6 /(k+2)(k+3)$ (see [8,11]).

Now let $\mathfrak{g}=s l_{n+1}(\mathbb{C})$. Then the $W$-algebra $W_{s l_{n+1}}(1, k)$ has the central charge

$$
c_{1, k}=\frac{k n(k+2 n+3)}{(k+n+1)(k+n+2)} .
$$

The simple VOA $L_{\hat{\mathfrak{g}}}(1,0)$ is isomorphic to the lattice VOA $V_{A_{n}}$. Since the lattice VOA $V_{A_{n}^{k+1}} \cong \bigotimes_{i=1}^{k+1} V_{A_{n}}$, we have the following proposition by induction on $k$.

Proposition 5.3. The lattice VOA $V_{A_{n}^{k+1}}$ contains a full subVOA isomorphic to

$$
W_{s l_{n+1}}(1,1) \otimes \cdots \otimes W_{s l_{n+1}}(1, k) \otimes L_{\hat{s l}_{n+1}}(k+1,0) .
$$

Combining this with Proposition 4.5, we have our second main result. 
Proposition 5.4. The parafermion VOA $K\left(s l_{k+1}, n+1\right)$ contains a full subVOA isomorphic to

$$
W_{s l_{n+1}}(1,1) \otimes \cdots \otimes W_{s l_{n+1}}(1, k)
$$

In particular, we obtain an isomorphism $K\left(s l_{2}, n+1\right) \cong W_{s l_{n+1}}(1,1)$ when $k=1$.

\section{ACKNOWLEDGMENTS}

The author thanks Feng Xu, Xiangyu Jiao, Hiromichi Yamada and Tomoyuki Arakawa for valuable discussions.

\section{REFERENCES}

[1] Daniel Altschüler, Michel Bauer, and Claude Itzykson, The branching rules of conformal embeddings, Comm. Math. Phys. 132 (1990), no. 2, 349-364. MR1069826 (91j:17032)

[2] Chongying Dong, Ching Hung Lam, and Hiromichi Yamada, $W$-algebras related to parafermion algebras, J. Algebra $\mathbf{3 2 2}$ (2009), no. 7, 2366-2403, DOI 10.1016/j.jalgebra.2009.03.034. MR2553685(2011b:17053)

[3] Chongying Dong, Ching Hung Lam, Qing Wang, and Hiromichi Yamada, The structure of parafermion vertex operator algebras, J. Algebra 323 (2010), no. 2, 371-381, DOI 10.1016/j.jalgebra.2009.08.003. MR2564844(2011a:17041)

[4] Chongying Dong and James Lepowsky, Generalized vertex algebras and relative vertex operators, Progress in Mathematics, vol. 112, Birkhäuser Boston Inc., Boston, MA, 1993. MR:1233387(95b:17032)

[5] Chongying Dong and Qing Wang, The structure of parafermion vertex operator algebras: general case, Comm. Math. Phys. 299 (2010), no. 3, 783-792, DOI 10.1007/s00220-010-11148. MR2718932 (2011h:17037)

[6] Igor Frenkel, James Lepowsky, and Arne Meurman, Vertex operator algebras and the Monster, Pure and Applied Mathematics, vol. 134, Academic Press Inc., Boston, MA, 1988. MR996026 (90h:17026)

[7] Igor B. Frenkel and Yongchang Zhu, Vertex operator algebras associated to representations of affine and Virasoro algebras, Duke Math. J. 66 (1992), no. 1, 123-168, DOI 10.1215/S00127094-92-06604-X. MR1159433 (93g:17045)

[8] P. Goddard, A. Kent, and D. Olive, Virasoro algebras and coset space models, Phys. Lett. B 152 (1985), no. 1-2, 88-92, DOI 10.1016/0370-2693(85)91145-1. MR778819 (86m:81092)

[9] Robert L. Griess Jr. and Ching Hung Lam, EE 8 -lattices and dihedral groups, Pure Appl. Math. Q. 7 (2011), no. 3, Special Issue: In honor of Jacques Tits, 621-743. MR.2848589 (2012i:11068)

[10] Koji Hasegawa, Spin module versions of Weyl's reciprocity theorem for classical Kac-Moody Lie algebras - an application to branching rule duality, Publ. Res. Inst. Math. Sci. 25 (1989), no. 5, 741-828, DOI 10.2977/prims/1195172705. MR1031225 (91f:17023)

[11] V. G. Kac and A. K. Raina, Bombay lectures on highest weight representations of infinitedimensional Lie algebras, Advanced Series in Mathematical Physics, vol. 2, World Scientific Publishing Co. Inc., Teaneck, NJ, 1987. MR1021978 (90k:17013)

[12] Victor G. Kac and Minoru Wakimoto, Modular and conformal invariance constraints in representation theory of affine algebras, Adv. in Math. 70 (1988), no. 2, 156-236, DOI 10.1016/0001-8708(88)90055-2. MR954660(89h:17036)

[13] Tomoki Nakanishi and Akihiro Tsuchiya, Level-rank duality of WZW models in conformal field theory, Comm. Math. Phys. 144 (1992), no. 2, 351-372. MR.1152377 (93a:81181)

[14] C. Pauly, Strange duality revisited, arXiv:1204.1186v1.

Institute of Mathematics, Academia Sinica, Taipei, Taiwan 10617 - And - National

Center for Theoretical Sciences, Taiwan

E-mail address: chlam@math.sinica.edu.tw 